\title{
REVIEW
}

\section{Weaning failure of cardiac origin: recent advances}

\author{
Jean-Louis Teboul*, Xavier Monnet, and Christian Richard \\ This article is one of ten reviews selected from the Yearbook of Intensive Care and Emergency Medicine 2010 (Springer Verlag) and co-published \\ as a series in Critical Care. Other articles in the series can be found online at http://ccforum/series/yearbook. Further information about the \\ Yearbook of Intensive Care and Emergency Medicine is available from http://www.springer.com/series/2855.
}

\section{Introduction}

Mechanical ventilation generally exerts negative hemodynamic effects in patients with normal cardiac function mainly because of the reduction in venous return induced by positive intrathoracic pressure at each insufflation [1]. By contrast, positive pressure ventilation exerts beneficial effects in patients with cardiogenic pulmonary edema such that it is routinely used as a therapy in this category of patients [2, 3]. Conversely, cardiac consequences of spontaneous breathing may be responsible for weaning failure in patients with left heart disease, even though the mechanical ventilation was required for respiratory failure of non-cardiac origin. Since its first description more than twenty years ago [4], cardiogenic pulmonary edema has been recognized as a frequent cause of weaning failure in patients with underlying left cardiac dysfunction.

In this chapter, we first briefly summarize the mechanisms by which pulmonary edema can develop during weaning. We then emphasize how weaning failure of cardiac origin can be detected at the bedside since significant progress has recently been made in this field. Finally, we describe the therapeutic options currently available.

\section{Mechanisms contributing to the development of weaning-induced pulmonary edema}

The mechanisms that contribute to development of cardiogenic pulmonary edema during weaning have been extensively detailed in a previous review [5]. These mechanisms are complex and mainly include the inspiratory fall in intrathoracic pressure, the increase in work of breathing, and the catecholamine discharge that occur during abrupt transfer from mechanical ventilation to spontaneous breathing [5]. Inspiratory fall in intrathoracic pressure tends to increase the systemic venous

${ }^{*}$ Correspondence: jean-louis.teboul@bct.aphp.fr Service of Medical Intensive Care, Centre Hospitalier de Bicêtre, 78 rue du Général Leclerc, 94270 Le Kremlin-Bicêtre, France

(c) Springer-Verlag Berlin Heidelberg 2010. This work is subject to copyright. All rights are reserved, whether the whole or part of the material is concerned, specifically the rights of translation, reprinting, reuse of illustrations, recitation, broadcasting, reproduction on microfilm or in any other way, and storage in data banks. Duplication of this publication or parts thereof is permitted only under the provisions of the German Copyright Law of September 9, 1965, in its current version, and permission for use must always be obtained from Springer-Verlag. Violations are liable for prosecution under the German Copyright Law. return pressure gradient and the central blood volume [5], and to decrease the left ventricular (LV) ejection pressure gradient with a resulting increase in LV afterload [5]. A marked increase in work of breathing may increase cardiac work and myocardial oxygen demand [5]. The increased adrenergic tone may also increase venous return, LV afterload, cardiac work, and myocardial oxygen demand and may thus potentially result in myocardial ischemia in predisposed patients $[4,6]$. In patients with pre-existing right ventricular (RV) disease, an increase in weaning-induced RV afterload may occur because of hypoxemia or worsening of intrinsic positive end-expiratory pressure (PEEPi) [5]. In addition to the simultaneous increase in systemic venous return, the increased RV afterload may lead to a marked RV enlargement during weaning, thus impeding the diastolic filling of the left ventricle through a biventricular interdependence mechanism [5]. In summary, elevation of the LV filling pressure can occur during weaning because of an increase in LV preload and/or decrease in LV compliance (myocardial ischemia, biventricular interdependence) and/or increase in LV afterload. However, in the absence of left heart disease, the rise in pulmonary artery occlusion pressure (PAOP) is limited [7, 8]. In contrast, marked increases in PAOP have been reported to occur during unsuccessful weaning in patients with left heart disease [4, 9-12], who can thus be suspected to have failed to wean because of the onset of cardiogenic pulmonary edema.

\section{Diagnosis of weaning-induced pulmonary edema Clinical context}

The diagnosis of weaning-induced pulmonary edema should be suspected when intolerance to a spontaneous breathing trial (SBT) occurs and other causes of weaning failure have been discarded. The suspicion is reinforced by the fact that the patient has a previous history of left heart disease. Patients with a combination of left heart disease 
and chronic obstructive pulmonary disease (COPD) are at higher risk of weaning-induced pulmonary edema. In this situation, the increase in airway resistance amplifies two mechanisms responsible for LV filling pressure elevation: 1) the fall in intrathoracic pressure is exaggerated at inspiration leading to a marked increase in LV afterload during spontaneous breathing; and 2) the work of breathing further augments leading to increased myocardial oxygen demand with inherent risks of myocardial ischemia in predisposed patients. In addition, the biventricular interdependence phenomenon can be marked in COPD patients with pre-existing RV dilation and thus can significantly contribute to LV filling pressure elevation.

The early onset of respiratory distress after starting a weaning trial is assumed to be suggestive of weaninginduced pulmonary edema, although there is no clear evidence in the literature to support this assumption. In our experience, the combined increase in arterial pressure and heart rate during unsuccessful weaning is quite suggestive of weaning failure of cardiac origin [11, 12], although false positive and false negatives can be encountered [11].

\section{Right heart catheterization}

Right heart catheterization can be helpful in the evaluation of acute dyspnea in patients with concomitant pulmonary and cardiac disease, since it allows measurement of PAOP, pulmonary artery pressure, right atrial pressure, and oxygen-derived variables [13]. In this regard, right heart catheterization was first proposed to establish the diagnosis of weaning-induced pulmonary edema. A higher than normal value of PAOP measured during an unsuccessful SBT is highly suggestive of weaning-induced pulmonary edema [14]. There is no definite value of PAOP above which cardiogenic pulmonary edema develops, although $18 \mathrm{mmHg}$ is recognized as a classical cut-off value [15]. Numerous studies have shown increases in PAOP during weaning in patients who failed to wean $[4$, 9-12]. Lemaire and colleagues [4] reported an average increase in transmural PAOP from 8 to $25 \mathrm{mmHg}$ in unsuccessful weaning trials in 15 patients with both COPD and left heart disease. Interestingly, after diuretic therapy, $60 \%$ of these difficult-to-wean patients could be suc cessfully weaned with no further increase in PAOP during weaning [4]. This strongly suggests that the weaninginduced elevation of PAOP played a major role in the difficulty to wean of these patients.

In all these studies, the increase in PAOP during unsuccessful weaning was not associated with a decrease in cardiac output $[4,10-12]$. In fact, the weaning process is quite similar to an exercise test such that an increase in cardiac output is expected to occur in response to the increased work of breathing and to the stress created by the abrupt transfer of the patient from mechanical ventilation to spontaneous breathing [16]. Patients with impaired cardiac function may fail to sufficiently increase cardiac output and oxygen transport in response to increased oxygen requirements. These patients may thus experience not only an increase in PAOP but also a decrease in mixed venous blood saturation $\left(\mathrm{SvO}_{2}\right)$ during weaning as reported in clinical studies [10-12]. Although the decrease in $\mathrm{SvO}_{2}$ is obviously not an indicator of cardiogenic pulmonary edema, it can identify weaning failure from cardiac origin as well as the elevation in PAOP can. As long as the central venous oxygen saturation $\left(\mathrm{ScvO}_{2}\right)$ reflects $\mathrm{SvO}_{2}$, simple central venous catheterization could thus be a tool to detect weaning failure from cardiac origin. Further studies are, however, needed to confirm this hypothesis.

To summarize, right heart catheterization can be helpful for diagnosing weaning failure from cardiac origin since it may not only identify patients with elevated PAOP during weaning but also provide important information about the mechanisms responsible for weaning-induced acute cardiac dysfunction. Nevertheless, pulmonary artery catheterization remains an invasive procedure [13]. Recent research studies have been undertaken to find less invasive tools for identifying weaning failure from cardiac origin.

\section{Transthoracic echocardiography}

Echocardiography has become a routine tool for evaluating the cardiovascular status in critically ill patients. It is now possible to estimate LV filling pressures using Doppler transmitral flow and Doppler tissue imaging variables [17]. Doppler transmitral flow allows the early (E) and late (A) peak diastolic velocities to be measured, and tissue Doppler imaging of the mitral annulus allows the early (Ea) peak mitral annulus diastolic velocity to be measured. In a series of 39 difficult-to-wean patients, Lamia and colleagues [12] tested the hypothesis that E/A and $\mathrm{E} / \mathrm{Ea}$ could be used to detect weaning-induced elevation defined by a PAOP $\geq 18 \mathrm{mmHg}$ during a SBT. The major finding of this study was that the combination of E/A > 0.95 and of E/Ea > 8.5 at the end of the SBT predicted a PAOP $\geq 18 \mathrm{mmHg}$ with good sensitivity $(82 \%)$ and specificity (91\%) while the cut-off values of E/ A alone and of E/Ea alone had weak specificity (68\% and $73 \%$, respectively). The study by Lamia and colleagues [12] thus provides evidence that a totally non-invasive method can identify patients with weaning-induced pulmonary edema. Moreover, as echocardiography is a valuable method to evaluate cardiac function at the bedside, it can also provide clinicians with important information about the main mechanisms responsible for cardiac dysfunction during weaning. However, echocardiography is an operator-dependent method that requires a long training period to ensure critical care 
physicians are skilled enough to use it properly. Other tools that can more simply detect weaning-induced pulmonary edema are, therefore, necessary.

\section{Cardiac biomarkers}

B-type natriuretic peptide (BNP) and N-terminal proBNP (NT-proBNP) are peptides synthesized by the ventricular myocytes in response to an increased myocardial stretch. Both systolic and diastolic dysfunction of the left ventricle can result in high circulating BNP and NTproBNP levels. In critically ill patients, such cardiac biomarkers are increasingly used as screening tools to rule out cardiac dysfunction $[18,19]$. Two recent studies addressed the question of whether BNP or NT-proBNP could be used to identify patients who fail to wean for cardiac reasons $[20,21]$. However, the results of these two studies are not straightforward. Mekontso-Dessap and colleagues showed that, before the first weaning attempt, the plasma BNP level was higher in patients with subsequent weaning failure [20]. The area under the receiver operating characteristic (ROC) curve for plasma BNP to predict weaning failure was $0.89 \pm 0.04$ and a cutoff value of $275 \mathrm{pg} / \mathrm{ml}$ was associated with the highest diagnostic accuracy (86\%). However, one may wonder whether it is really pertinent to predict results of a simple and safe test such as a SBT, rather than performing it. Indeed, simply observing how a patient breathes during an unsuccessful SBT can provide a large amount of information about the mechanisms responsible for the weaning failure. Therefore, in our opinion, there is no real need to measure the BNP level simply in order to avoid performance of a SBT. Moreover, the fact that plasma BNP concentration was higher before the SBT in patients who subsequently failed the SBT does not mean that cardiac dysfunction and cardiogenic pulmonary edema occurred during the SBT. High baseline plasma BNP concentration before the SBT may rather reflect a more severe global condition in patients who did not tolerate the SBT. It should be stressed that the plasma BNP level can be elevated in cases of advanced age, sepsis, renal dysfunction, and pulmonary hypertension, even in the absence of left heart dysfunction [18]. In this context, systolic cardiac dysfunction assessed by echocardiography did not differ between patients who succeeded and patients who failed the weaning trial in the study by Mekontso-Dessap and colleagues [20]. Moreover, there was no difference in the BNP level before and at the end of the weaning trial in the two groups of patients. Overall the results reported by MekontsoDessap and colleagues [20] underline the uncertainty of using plasma BNP levels to reliably identify patients who experience weaning failure of cardiac origin. The study by Grasso and colleagues [21] evaluated the significance of NT-proBNP in detecting weaning failure of cardiac origin in COPD patients. The cardiac origin of weaning failure was determined by one cardiologist and one critical care physician blinded to the results of the NT-proBNP measurements [21]. Their diagnosis was based on the review of echocardiograms, electrocardiograms (EKGs), and clinical, hemodynamic, gas exchange and respiratory variables obtained at baseline and at the end of the SBT. Interestingly, the elevation of NT-proBNP during a SBT but not the baseline NT-proBNP predicted weaning-induced cardiac dysfunction with an acceptable accuracy [21]. Because of the limited number of patients included in this study, confirmation in a larger cohort of patients is needed.

Clearly, suspicion of acute cardiac dysfunction during weaning using a change in NT-proBNP levels should prompt further cardiac evaluation (e.g., echocardiography) aimed at confirming the cardiac origin of weaning failure. In our opinion, the divergent results reported by Mekontso-Dessap and colleagues [20] and Grasso and colleagues [21] should urge caution in the use of natriuretic peptide values for diagnosing weaninginduced pulmonary edema. It must be stressed that in neither of these two studies was pulmonary artery catheterization performed to evidence elevation of PAOP during weaning. Further studies are thus mandatory.

\section{Detection of weaning-induced hemoconcentration}

Weaning-induced pulmonary edema is assumed to be a hydrostatic pulmonary edema resulting from an increased LV filling pressure. Hydrostatic pulmonary edema is accompanied by transfer of a hypo-oncotic fluid from the lumen of the pulmonary capillaries toward the interstitium [22]. When the amount of transferred fluid is large enough, hydrostatic pulmonary edema may result in hemoconcentration that could be detected on the basis of changes in plasma protein or hemoglobin concentrations or hematocrit [22] (Figure 1). In a recent study, we hypothesized that an acute occurrence of hemoconcentration during weaning could help to diagnose weaning-induced pulmonary edema [11]. We defined weaning-induced pulmonary edema as intolerance to spontaneous breathing and elevation of PAOP above $18 \mathrm{mmHg}$ at the end of a SBT. We inserted a pulmonary artery catheter in 46 patients who failed two consecutive SBTs although there was no obvious cause of weaning failure [11]. Twenty-four of these patients experienced weaning-induced pulmonary edema with an increase in the median value of PAOP from $13 \mathrm{mmHg}$ (range: 7$16 \mathrm{mmHg}$ ) to $26 \mathrm{mmHg}$ (range: $18-50 \mathrm{mmHg}$ ) during the third SBT [11]. In these patients, the plasma protein concentration increased significantly during the SBT. An increase in plasma protein concentration greater than $6 \%$ during the weaning trial enabled weaning-induced pulmonary edema to be detected with a sensitivity of $87 \%$ 


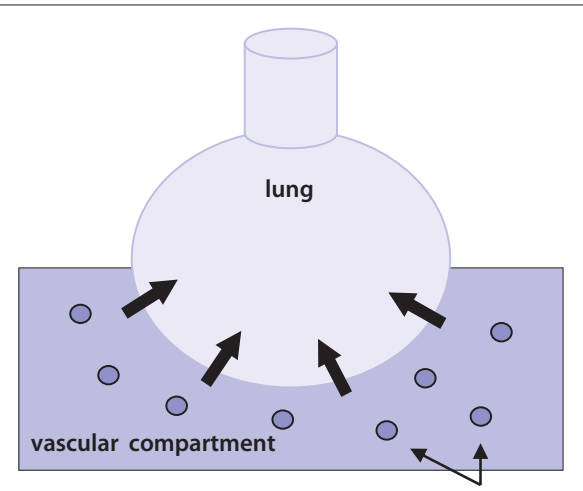

(a)

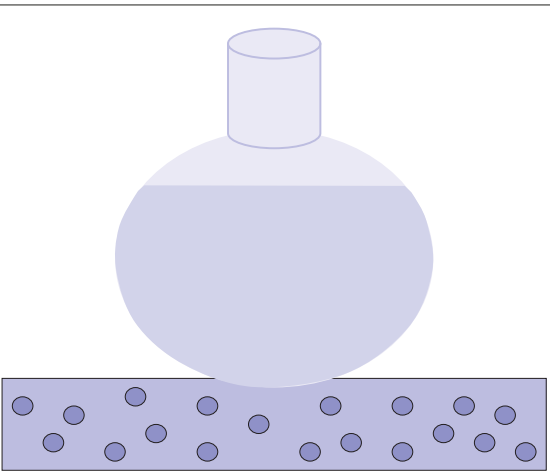

(b)

Figure 1. Consequences of fluid transfer during hydrostatic pulmonary oedema. (a) As weaning-induced pulmonary edema is of a hydrostatic nature, its development is characterized by transfer of a hypo-oncotic fluid from the pulmonary capillary lumen toward the interstitial compartment and then the alveoli. Molecules like proteins cannot flow across the lung barrier owing to their high molecular weight. (b) After equilibrium is reached, weaning-induced pulmonary edema is thus characterized by a contraction of the vascular compartment and an augmentation in the plasma protein concentration.

and a specificity of $95 \%$ [11]. This $6 \%$ cut-off provided a very high positive likelihood ratio value (19.25) and a negative likelihood ratio value of 0.13 [11]. The area under the ROC curve generated for changes in plasma protein concentration $(0.93 \pm 0.04)$ was significantly higher than that generated for changes in $\mathrm{SvO}_{2}$ during the SBT $(0.70 \pm 0.08)$ [11]. Interestingly, in 13 patients who experienced weaning-induced pulmonary edema at the third SBT, the fourth weaning trial was again monitored with a pulmonary artery catheter after they had received diuretics and/or vasodilators [11]. None of these patients experienced recurrent weaning-induced pulmonary edema and the plasma protein concentration did not change during their fourth SBT [11]. This study strongly suggests that measuring acute changes in plasma protein concentration during a weaning trial represents a minimally invasive alternative to right heart catheterization for identifying patients that experience weaninginduced pulmonary edema.

No study has compared the different tools aimed at diagnosing the cardiac origin of weaning failure. In our opinion, measuring the change in plasma protein concentration during a SBT is the simplest tool for reliably screening patients for weaning-induced pulmonary edema. Echocardiography can provide further confirmation, and can also offer useful information about the mechanisms responsible for the weaning failure.

\section{Therapeutic options}

The treatment of weaning-induced pulmonary edema should obviously take into account the mechanism suspected to be mainly responsible for the weaning failure. It is thus important to first carefully analyze the cardiovascular response to a SBT and then to monitor the chosen therapy using an invasive or non-invasive hemodynamic tool.
Diuretic therapy must be considered when excessive increased preload during weaning is suggested as the main mechanism responsible for weaning failure. In this context, in the study by Lemaire and colleagues [4], 9 of the 15 patients who initially failed to wean because of development of pulmonary edema successfully weaned after one week of treatment with furosemide, which resulted in fluid losses equivalent to 5 litres. After treatment, PAOP was lower at the end of a SBT than it was before treatment was administered ( $9 \pm 3$ vs $25 \pm 15 \mathrm{mmHg}$ ). The attitude of empirically giving diuretics in every difficult-to-wean patient has become more and more frequent. Nevertheless, it seems to us difficult to recommend such an approach since extra-cardiac causes are responsible for weaning failure in at least $50 \%$ of this group of patients $[11,12]$ and since uncontrolled diuretic therapy can have potentially harmful effects.

In cases where excessive increased afterload is suspected as the main mechanism, administration of vasodilators may be chosen instead of (or in addition to) diuretics. In our experience, the onset of a marked increase in systolic arterial pressure during a SBT represents a reasonable indication for vasodilator administration. Nitrates may be a good therapeutic choice since this treatment can decrease both LV afterload and central blood volume (cardiac preload effect). In addition, because of its coronary vasodilating effects, it can be even more helpful when myocardial ischemia is one of the mechanisms responsible for the weaning failure.

Use of $\beta_{1}$-agonist agents, such as dobutamine, is not logical in this context since weaning-induced pulmonary edema can hardly be caused by a reduction in cardiac contractility (see above). Moreover, unsuccessful weaning is associated with a huge increase in endogenous catecholamine discharge and administration of $\beta_{1}$-agonist 
agents can further increase myocardial oxygen demand with its inherent risks of myocardial ischemia in predisposed patients. However, phosphodiesterase inhibitors, such as enoximone, have been reported to be efficacious for treating the weaning-induced pulmonary edema that can develop after cardiac surgery [9]. It is likely that the vasodilating effect of these drugs could have significantly contributed to their beneficial impact. Finally, calcium channel blockers have been reported to facilitate successful weaning in the particular context of hypertrophic cardiomyopathy [23].

In terms of ventilatory modalities, there is no definite recommendation. After unsuccessful weaning of cardiac origin, reinstitution of mechanical ventilation is mandatory. The practice of progressive decrements of pressure support levels while keeping 5 to $8 \mathrm{cmH}_{2} \mathrm{O}$ of PEEP could be an interesting option since pressure support is assumed to increase LV afterload less than spontaneous breathing [24]. After extubation, non-invasive positive pressure ventilation using a face mask could be used. However, there is no definite recommendation about this practice [25].

\section{Conclusion}

Acute cardiac dysfunction and cardiogenic pulmonary edema may occur during weaning from mechanical ventilation, especially in patients with a history of left heart disease and COPD. Among the complex and intricate mechanisms, myocardial ischemia, excessive increased LV afterload, and increased cardiac preload play predominant contributing roles. Measuring the elevation in PAOP using right heart catheterization was first proposed as a means of diagnosing weaning failure of cardiac origin. Less invasive tools, such as transthoracic echocardiography or measurements of plasma protein concentration, have recently been proposed as valuable alternative diagnostic methods for weaninginduced pulmonary edema. There is no codified treatment for weaning-induced pulmonary edema. Use of diuretics and/or nitrates should be considered after careful analysis of the main contributing mechanisms.

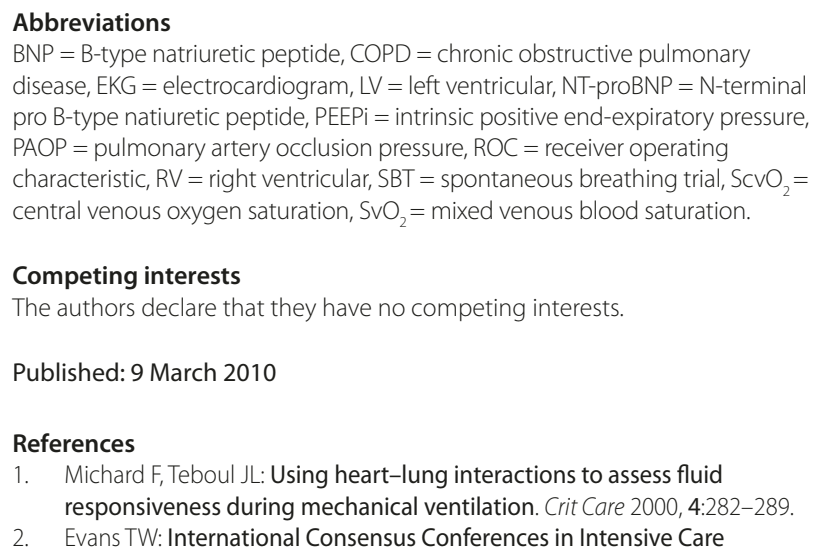

Published: 9 March 2010

\section{References}

1. Michard F, Teboul $\mathrm{J}$ : Using heart-lung interactions to assess fluid responsiveness during mechanical ventilation. Crit Care 2000, 4:282-289.

2. Evans TW: International Consensus Conferences in Intensive Care

Medicine: non-invasive positive pressure ventilation in acute respiratory failure. Intensive Care Med 2001, 27:166-178.

3. Winck JC, Azevedo LF, Costa-Pereira A, Antonelli M, Wyatt JC: Efficacy and safety of non-invasive ventilation in the treatment of acute cardiogenic pulmonary edema-a systematic review and meta-analysis. Crit Care 2006, 10:R69.

4. Lemaire F, Teboul $J \mathrm{~L}$, Cinotti $L$, et al:: Acute left ventricular dysfunction during unsuccessful weaning from mechanical ventilation. Anesthesiology 1988, 69:171-179.

5. Lamia B, Monnet X, Teboul JL: Weaning-induced cardiac dysfunction. In (1 edition) Yearbook of Intensive Care and Emergency Medicine. Springer, Heidelberg, 2005:239-245.

6. Chatila W, Ani S, Cuaglianone D, Jacob B, Amoateng-Adjepong Y, Manthous $\mathrm{CA}$ : Cardiac ischemia during weaning from mechanical ventilation. Chest 1996, 109:577-583.

7. Teboul $J$, Abrouk F, Lemaire F: Right ventricular function in COPD patients during weaning from mechanical ventilation. Intensive Care Med 1988, 14:483-485.

8. De Backer D, Haddad PE, Preiser JC, Vincent JL: Hemodynamic responses to successful weaning from mechanical ventilation after cardiovascular surgery. Intensive Care Med 2000, 26:1201-1206.

9. Paulus S, Lehot JJ, Bastien O, Piriou V, George M, Estanove S: Enoximone and acute left ventricular failure during weaning from mechanical ventilation after cardiac surgery. Crit Care Med 1994, 22:74-80.

10. Jubran A, Mathru M, Dries D, Tobin MJ: Continuous recordings of mixed venous oxygen saturation during weaning from mechanical ventilation and the ramifications thereof. Am J Respir Crit Care Med 1998, 158:1763-1769.

11. Anguel N, Monnet X, Osman D, Castelain V, Richard C, Teboul JL: Increase in plasma protein concentration for diagnosing weaning-induced pulmonary oedema. Intensive Care Med 2008,34:1231-1238.

12. Lamia B, Maizel J, Ochagavia A, et al.: Echocardiographic diagnosis of pulmonary artery occlusion pressure elevation during weaning from mechanical ventilation. Crit Care Med 2009, 37:1696-1701.

13. Payen $D$, Gayat $E$ : Which general intensive care unit patients can benefit from placement of the pulmonary artery catheter? Crit Care 2006, 10(Suppl 3):S7.

14. Richard C, Teboul $\mathrm{J}$ : Weaning failure from cardiovascular origin. Intensive Care Med 2005, 31:1605-1607.

15. McHugh TJ, Forrester JS, Adler L, Zion D, Swan HJ: Pulmonary vascular congestion in acute myocardial infarction: hemodynamic and radiologic correlations. Ann Intern Med 1972, 76:29-33.

16. Pinsky MR: Breathing as exercise: the cardiovascular response to weaning from mechanical ventilation. Intensive Care Med 2000, 26:1664-1666.

17. Vignon $\mathrm{P}$, AitHssain A, Francois B, et al.: Echocardiographic assessment of pulmonary artery occlusion pressure in ventilated patients: a transoesophageal study. Crit Care 2008, 12:R18.

18. McLean AS, Huang SJ, Salter M: Bench-to-bedside review: the value of cardiac biomarkers in the intensive care patient. Crit Care 2008, 12:215.

19. Coquet I, Darmon M, Doise JM, et al:: Performance of N-terminal-pro-B-type natriuretic peptide in critically ill patients: a prospective observational cohort study. Crit Care 2008, 12:R137.

20. Mekontso-Dessap A, de Prost N, Girou E, et al:: B-type natriuretic peptide and weaning from mechanical ventilation. Intensive Care Med 2006, 32:1529-1536.

21. Grasso S, Leone A, De Michele M, et al.: Use of N-terminal pro-brain natriuretic peptide to detect acute cardiac dysfunction during weaning failure in difficult-to-wean patients with chronic obstructive pulmonary disease. Crit Care Med 2007, 35:96-105.

22. Figueras J, Weil MH: Increases in plasma oncotic pressure during acute cardiogenic pulmonary edema. Circulation 1977, 55:195-199.

23. Adamopoulos C, Tsagourias M, Arvaniti K, Veroniki F, Matamis D: Weaning failure from mechanical ventilation due to hypertrophic obstructive cardiomyopathy. Intensive Care Med 2005, 31:734-737.

24. Richard C, Teboul JL, Archambaud F, Hebert JL, Michaut P, Auzepy P: Left ventricular function during weaning of patients with chronic obstructive pulmonary disease. Intensive Care Med 1994, 20:181-186.

25. Antonelli M, Pennisi MA, Montini L: Noninvasive ventilation in the clinical setting-experience from the past 10 years. Crit Care, 2005 9:98-103.

doi:10.1186/cc8851

Cite this article as: Teboul $J-L$, et al:: Weaning failure of cardiac origin: recent advances. Critical Care 2010, 14:211. 